\title{
Overtreatment by Misdiagnosis of Pseudoinvasion in TLH
}

\author{
Junko Wroblewski, MD, PhD, Hidehiro Kawagoe, MD, PhD, Hiroki Nasu, MD, \\ Kyoko Kawamura, MD, PhD, Yoshihiko Tashiro, MD, PhD, Greggory Wroblewski, BS, MA, \\ Naofumi Okura, MD, PhD
}

Department of Obstetrics and Gynecology (Drs J. Wroblewski, Kawagoe, Nasu, Kawamura, and Okura) and Pathology (Dr Tashiro), National Hospital Organization Kokura Medical Center, Kitakyushu, Japan.

Division of Functional Neuroanatomy, Yamaguchi University Graduate School of Medicine, Ube, Japan (G. Wroblewski).

\begin{abstract}
Introduction: Despite reports of pseudo lymphovascular space involvement (LVSI) in total laparoscopic hysterectomy (TLH) in recent years, we recently experienced a misdiagnosis of pseudo-LVSI after TLH for uterine myoma, having observed irregularities in excised specimens. Additional surgery found no abnormalities, resulting in an unfortunate case of overtreatment. For this reason, we reviewed cases of TLH for benign uterine disorders performed at our hospital for the presence of similar pseudoinvasion.
\end{abstract}

Case Description: We re-examined 53 cases for the presence of intravascular endometrial tissue from patients who had undergone TLH for benign uterine disorders. In a 42-year-old patient who had undergone TLH for uterine myoma, we found a small amount of complex atypical endometrial hyperplasia and observed intravascular agglomerations of atypical endometrial cells at multiple sites, leading to a diagnosis of LVSI. We performed additional surgery (laparotomy), but findings were unremarkable. Pseudo-LVSI was identified in 8 of 53 cases (15.1\%): in 2 of 21 (9.5\%) operations performed with the ClearView uterine manipulator and in 6 of 32 (18.8\%) performed with the Vcare uterine manipulator.

Discussion: Differentiation between "true" LVSI and grossing artifacts remains difficult, and a noteworthy case of overtreatment such as this highlights the need to reinstitute differentiation as a salient topic of discussion among surgeons and pathologists. Similarly, the existence of pseudoinvasion in a significant number of the retrospectively reviewed cases, in light of its still-undetermined clinical significance, is an interesting finding that warrants additional investigation to avoid both overtreatment and undertreatment of such cases.

Key Words: Laparoscopic hysterectomy, LVSI, Misdiagnosis, Overtreatment, Pseudoinvasion.

Citation Wroblewski J, Kawagoe H, Nasu H, Kawamura K, Tashiro Y, Wroblewski G, Okura N. Overtreatment by misdiagnosis of pseudoinvasion in TLH. CRSLS e2015.00103. DOI: 10.4293/CRSLS.2015.00103.

Copyright (c) 2016 by SLS, Society of Laparoendoscopic Surgeons. This is an open-access article distributed under the terms of the Creative Commons Attribution-Noncommercial-ShareAlike 3.0 Unported license, which permits unrestricted noncommercial use, distribution, and reproduction in any medium, provided the original author and source are credited.

Address correspondence to: Junko Wroblewski, MD, Department of Obstetrics and Gynecology, National Hospital Organization Kokura Medical Center, 10-1

Harugaoka, Kokuraminami-ku, Kitakyusyu-shi, Fukuoka, 802-8533, Japan. Telephone: 81-93-921-8881, Fax: 81-93-921-5072, E-mail: junkou@kokura2.hosp.go.jp.

\section{INTRODUCTION}

In the past 2 decades, total laparoscopic hysterectomy (TLH) has become an increasingly popular surgical method, first for benign uterine disorders and more recently for malignancies of the uterine corpus. ${ }^{1-3}$ In recent years, centers throughout Japan have begun to adopt the procedure in a great many cases, ${ }^{4-6}$ including our hospital where we perform TLH with a uterine manipulator.
In a recent case, we found a small amount of complex atypical endometrial hyperplasia in an excised specimen from a patient in whom TLH was performed after preoperative diagnosis of benign uterine myoma. In the specimen, agglomerations of atypical endometrial cells were observed inside blood vessels of the deep muscle layer of the uterus at multiple sites, and it was originally deemed a likely case of lymphovascular space invasion (LVSI). For that reason, after explaining to the patient 
that malignancy could not be ruled out, we conducted a laparotomy of the inside of the pelvis at her request the following day. However, histopathological examination was unremarkable, and cells from the intravascular tissue were ultimately deemed to be free of abnormalities by several pathologists. Thus, this example of pseudoinvasion resulted in an unfortunate case of overtreatment.

Among top pathologists, it is widely known that vascular pseudoinvasion can often be recognized in specimens from TLH or laparoscopically assisted vaginal hysterectomy (LAVH) and that correct identification may prevent unnecessary additional surgery..$^{7-11}$ Despite this fact, however, this topic is mostly absent from discussion by obstetrician-gynecologists at meetings and conferences on clinical practice in Japan, and our search of the literature did not reveal any reports reflecting upon or addressing this diagnostic stumbling block in the present context.

In the current study, we report our retrospective review of 53 cases in which TLH was conducted at our hospital following benign uterine disorder diagnosis to determine whether similar pseudoinvasion was present in those specimens as well. We also discuss the difficulty in distinguishing between pseudoinvasion and genuine LVSI and highlight issues that have been identified in the current international literature. Our findings underscore a need for further research on how best to mitigate the risk of both undertreatment and overtreatment in similar cases in the future.

\section{MATERIALS AND METHODS}

\section{Index Case}

The case that led to the formulation of this study involved a 42-year-old patient with a body mass index of 23.1 who presented with a primary complaint of heavy menstrual bleeding. Magnetic resonance imaging (MRI) revealed multiple submucosal and subserosal myomas as well as a left ovarian cystic benign tumor. Papanicolaou tests of the uterine cervix and endometrium were both negative. Her preoperative diagnosis was uterine myoma and left benign ovarian tumor, for which we performed TLH and left salpingo-oophorectomy with a Vcare uterine manipulator (ConMed, Utica, New York, USA).

In one section of excised specimen, abnormal endometrial tissue was found in a focused, limited area (Figure 1A), and histological examination revealed complex atyp-

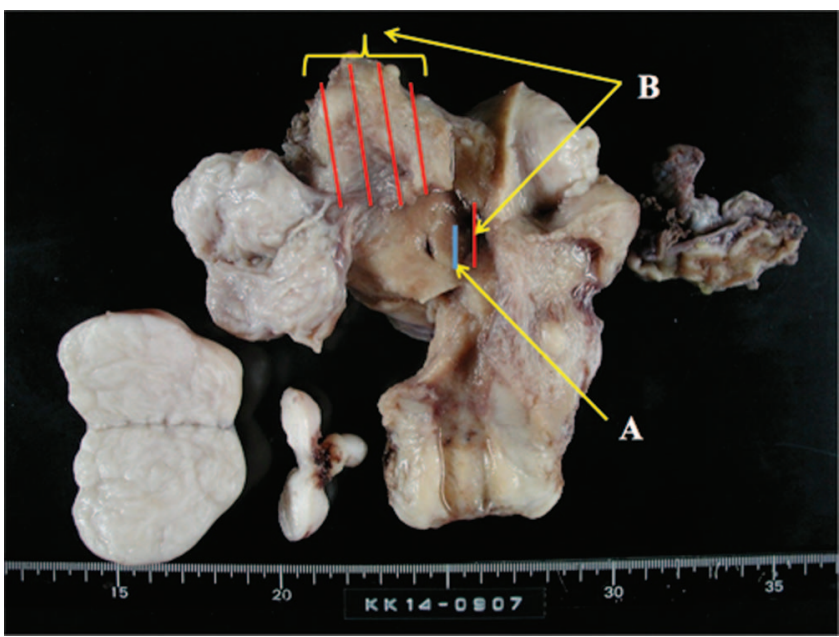

Figure 1. View of the entire uterus. (A) Complex atypical endometrial hyperplasia was observed in the area outlined in blue. (B) Agglomerations of atypical endometrial cells inside of vascular spaces were observed in the outer half of the myometrium and are outlined in red.

ical endometrial hyperplasia (Figure 2A). In multiple specimens from the outer half of the myometrium (Figure 1B), agglomerations of atypical cells and stromal tissue were observed in the vascular space (Figure $2 \mathbf{B}, \mathbf{D}$ ). Endometrial gland fragments and inflammatory debris were also seen (Figure 2C). The vascular epithelium at sites where atypical cells were observed was CD34 (endothelial marker) positive (Figure 3A) and D2-40 (lymphatic endothelial marker) negative (Figure 3B) and was judged to have invaded the vascular space. Although we had previously observed minor debris in various spaces in the uterine muscle in specimens from prior patients, the extent and amount of agglomerations and debris in the index case, in combination with the presence of complex atypical endometrial hyperplasia, made the current case especially troubling. Thus, initial review deemed this a likely case of lymphovascular invasion.

We ordered further independent review of specimens by several specialist gynecologic pathologists to rule out the possibility of malignancy and to determine whether the present case was an example of true LVSI. However, the final pathology results took several weeks to be reported, and because the patient and her family had still not received pathology results for what was originally thought to be a benign disorder, they grew concerned and requested an immediate consultation. We presented the initial findings-the presence of complex atypical endometrial hyperplasia and atypical cells in the vascular space of the deep uterine muscle-and explained that uterine 

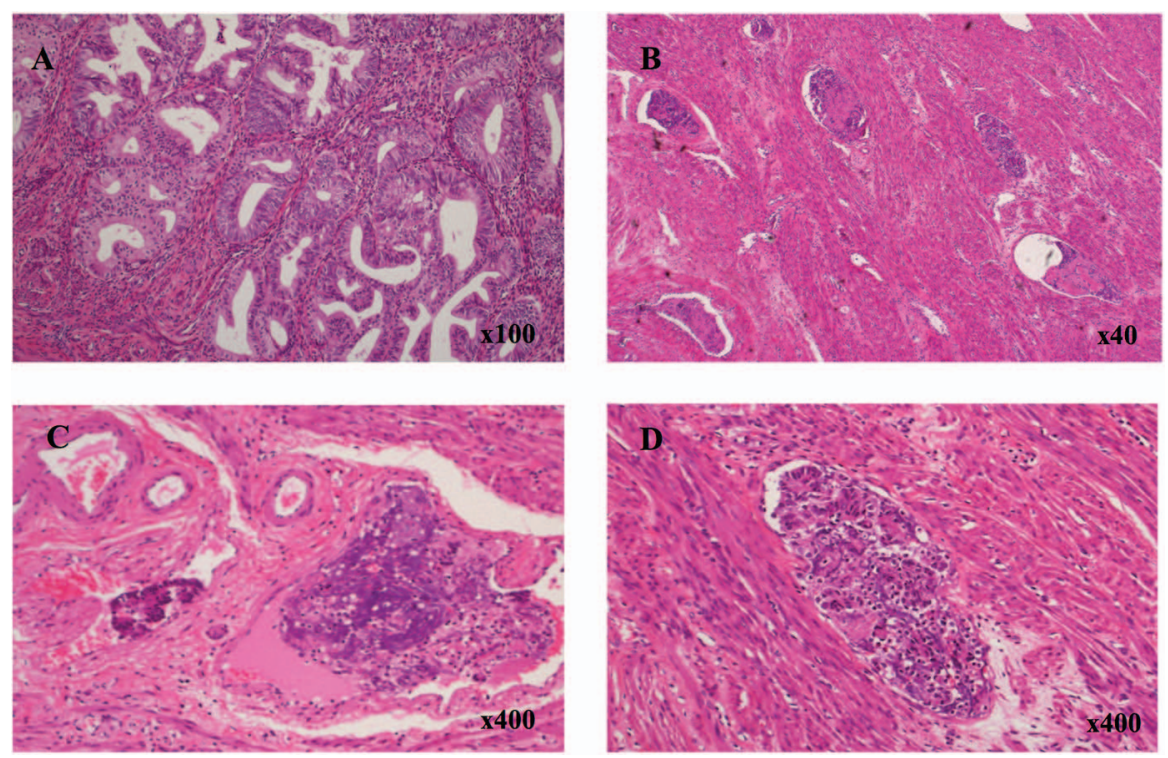

Figure 2. (A) Tissue sections from the complex atypical endometrial hyperplasia described in Fig. 1A. (B) Multiple agglomerations of atypical cells inside the vascular space. (C) Endometrial gland fragments and endometrial cell clusters, as well as inflammatory debris. (D) Vascular pseudoinvasion with endometrial glands and stromal tissue.
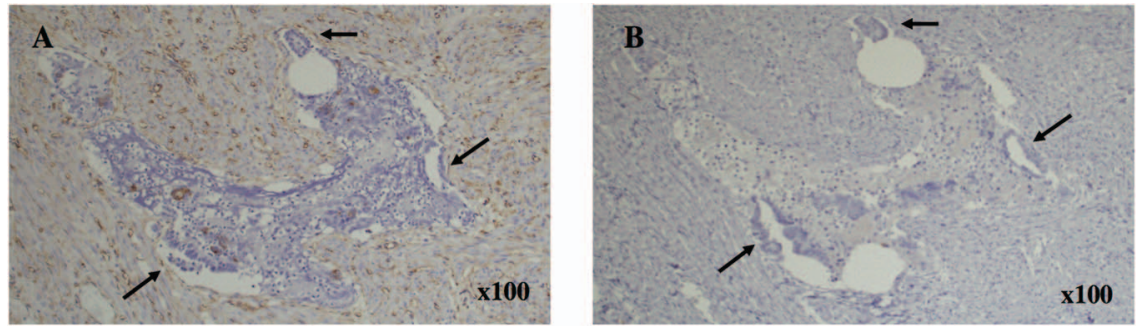

Figure 3. (A) Endometrial tissue in the vascular space (arrows), visualized with CD34 (endothelial marker) staining. (B) Paucity of D2-40 (lymphatic endothelial marker)-stained space around the endometrial tissue (arrows).

corpus cancer and LVSI could not be entirely ruled out. Subsequent computed tomographic (CT) scan and MRI did not reveal any metastases. Although given the option of continued observation without surgery, the patient and her family requested additional histopathological confirmation without further delay, this time via laparotomy instead of laparoscopy. The former was requested to facilitate a more thorough and immediate inspection of the affected areas the second time around. We consequently conducted the additional surgery, in which washing cytology of the abdominal cavity, right salpingo-oophorectomy, pelvic lymphadenectomy, and omental biopsy were performed in the process. Peritoneal cytology and lymph node metastases were both negative, and the right adnexa did not have any abnormalities. By that time, a thorough examination of specimens by multiple pathologists had been completed, and cells from the tissue observed in the intravascular space were ruled unremarkable. The case was ultimately judged to be an example of vascular pseudoinvasion of normal uterine endometrium, and in the end, the additional surgery was unnecessary.

\section{Case Selection}

To determine whether similar observations might be present in specimens from other cases in which TLH was performed for benign disorders of the uterus at our hospital, we re-examined 53 cases in which TLH had been performed for such cases from 2012 through 2014. In addition to the existing slides, we made 4 to 9 additional slides (an average of 6 ) for each case, and 3 of the authors (J.W., K.K., Y.T.) examined them for the presence of endometrial tissue in the lymphovascular space. Written permission to use the patients' specimens for research 

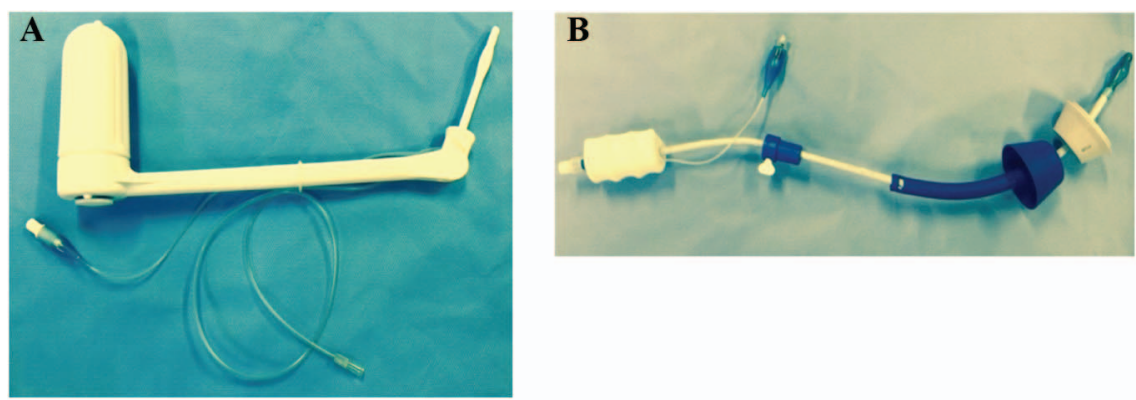

Figure 4. (A) ClearView and (B) Vcare uterine manipulators. In both, the balloons are filled with $5 \mathrm{ml}$ of air.

Table 1.

Patient characteristics ${ }^{\mathrm{a}}$

\begin{tabular}{lll}
\hline $\begin{array}{l}\text { Patient and Surgery } \\
\text { Characteristics }\end{array}$ & Total Group & $\begin{array}{l}\text { Pseudo-LSVI } \\
\text { Group }\end{array}$ \\
\hline $\begin{array}{l}\text { Patients, n } \\
\text { Age range, years (median) }\end{array}$ & $33-65(45.5)$ & $8(15.1)^{\mathrm{c}}$ \\
$\begin{array}{l}\text { Body mass index range } \\
\quad \text { median) }\end{array}$ & $16.1-31.6(22.7)$ & $18.2-30.8(24.3)$ \\
$\quad \begin{array}{l}\text { Uterine myoma } \\
\text { Adenomyosis }\end{array}$ & $39(73.6)^{\mathrm{c}}$ & $6(15.3)^{\mathrm{d}}$ \\
$\begin{array}{l}\text { Simple endometrial } \\
\quad \text { hyperplasia }\end{array}$ & $12(22.6)$ & $2(16.6)$ \\
CIN2 & $1(1.9)$ & 0 \\
Uterine manipulator & & 0 \\
ClearView & $21(39.6)$ & $2(9.5)$ \\
$\quad$ Vcare & $32(60.3)$ & $6(18.8)$ \\
$\quad$ Total & 53 & 8 \\
\hline
\end{tabular}

${ }^{\text {a }}$ The index case is not included.

${ }^{\mathrm{b}}$ Diagnosis of invasion of (pseudo) benign endometrial tissue into the lymphovascular space (LSVI).

${ }^{\mathrm{C}}$ Number of patients (percentage of total group of 53).

${ }^{\mathrm{d}}$ Number of patients in described subgroup (percentage of total patients in the subgroup).

purposes was obtained during the presurgical intake interview, and Institutional Review Board approval was not required because of the retrospective nature of this study.

In the current study, we used 2 different kinds of uterine manipulator: the ClearView (Clinical Innovation, LLC, Salt Lake City, Utah, USA) (Figure 4A) from April 2012 through October 2013, and the Vcare (ConMed) (Figure 4B), from November 2013 through 2014. The reason for changing models was that operability was found to be superior with the Vcare model.

\section{RESULTS}

Details of the 53 cases are listed in Table $\mathbf{1}$.

In 8 of the 53 cases (15.1\%), endometrial gland and stromal tissue were confirmed in the lymphovascular space (pseudo-LVSI). Broken down by category, 6 of 39 (15.3\%) were uterine myoma with 2 of $12(16.7 \%)$ being adenomyosis. Twenty-one cases were performed with the ClearView uterine manipulator, and 2 (9.5\%) showed pseudoLVSI. Of the 32 operations that were performed with the Vcare uterine manipulator, 6 (18.8\%) identified pseudoLVSI. In cases in which Vcare was used (pseudo) benign endometrial gland vascular involvement was observed at more than twice the rate of those in which the ClearView was used.

Figure 5 shows representative examples of pseudo-LVSI observed in specimens from the aforementioned 8 cases. In some spaces, clumps of suspicious stromal tissue (Figure 5A) and atypical-appearing cells (Figure 5B) were found. Pronounced and clearly visible examples of endometrial tissue involvement in the outer half of the myometrium were routinely present (Figures $5 \mathbf{C}-\mathbf{E}$ ), and endometrial cells and debris were often observed in large arteries as well (Figure 5F).

\section{DISCUSSION}

The retrospective nature of the current study made distinguishing between genuine and pseudoinvasion relatively easy. All of the samples under review were taken from cases of benign disorders, thus readily discounting the possibility of malignancy for the 8 cases that displayed vascular involvement. In practice, however, distinguishing between the two is much more difficult. Regarding the importance of making an accurate distinction between pseudo- and true involvement, in a 2008 study entitled "Vascular pseudoinvasion in laparoscopic hysterectomy specimens: a diagnostic pitfall," Logani et al7 urged cau- 

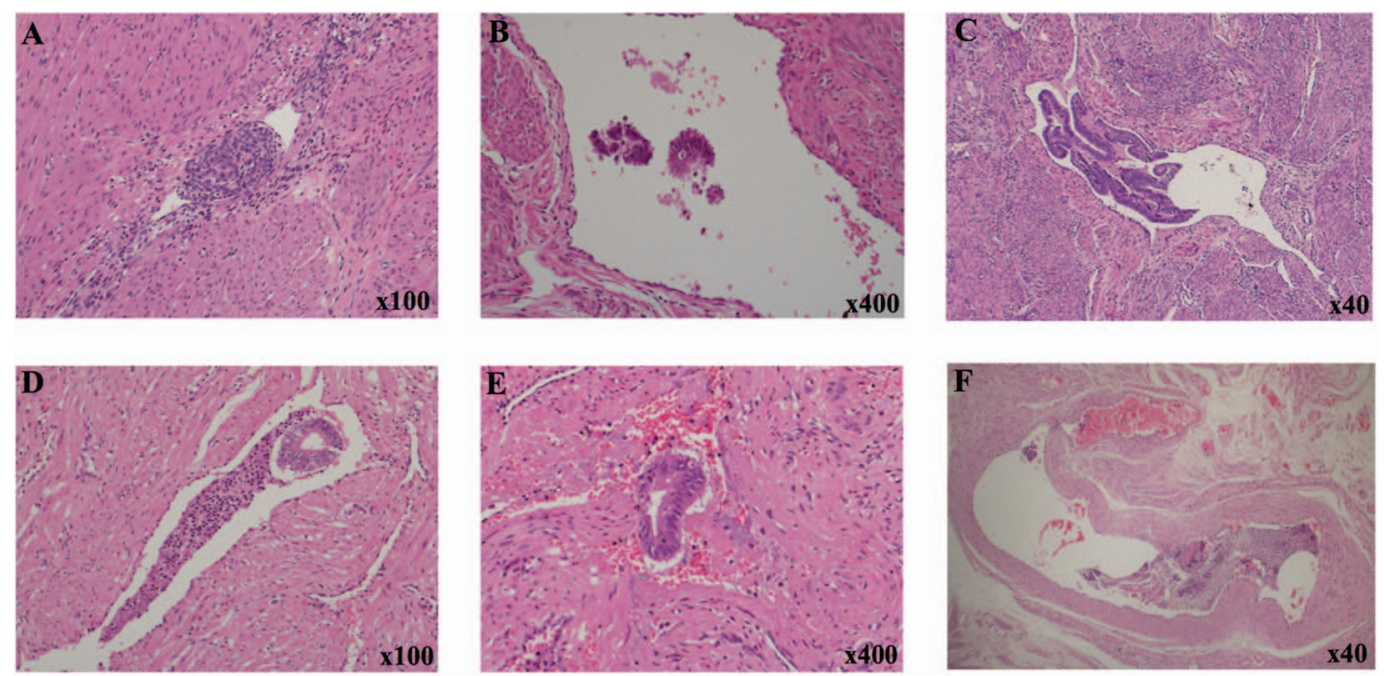

Figure 5. (A) Suspected vascular space involvement. (B) Atypical-appearing cells in the lymphovascular space. (C) Involvement of endometrial tissue in the outer half of the myometrium. (D) Endometrial gland and inflammatory tissue in lymphovascular space. (E) Endometrial gland in the vascular space in the outer half of the myometrium. (F) Small endometrial cells and debris in the large artery.

tion to avoid misinterpretation of pseudoinvasion as pathology. They observed an artifact resulting from the mechanical transportation of endometrial tumor tissue and benign endometrial glands and stroma into vascular channels in hysterectomy specimens performed with a uterine manipulator system. Through inflation of the manipulator's balloon, intrauterine pressure increased, resulting in transportation of endometrial tissue into the vascular space. As a possible solution, the spread of endometrial tissue and cells to the abdominal cavity and intrapelvic space via the fallopian tubes can be mitigated by ligating the fallopian tubes on both sides, resulting in an increasingly closed system that allows for a more easily achieved increase in intrauterine pressure.

In the aforementioned study, Logani et $\mathrm{al}^{7}$ also re-examined slides from 30 cases that were originally classified as having benign uterine pathology and reported that nonneoplastic endometrium was observed in the vascular space in 4 of $30(13 \%)$ of the cases. Our finding, in which the same was observed in 8 of 53 cases (15.1\%), is consistent with their previous results. Logani et al also emphasized the danger inherent in the misdiagnosis of vascular pseudoinvasion, as the existence (or lack thereof) of LVSI will greatly influence management of the condition during postsurgical supplemental treatment and subsequent clinical practice.

Adding to the importance of making the accurate distinction, LVSI has recently been recognized as an important independent prognostic factor. Weinberg et al ${ }^{12}$ cited LVSI as an isolated poor prognostic factor for recurrence and survival among women with intermediate-to-high-risk early-stage endometrial cancer, the only significant and consistent one of its kind. In additional, Guntupalli et al ${ }^{13}$ and Nofech-Mozes et $\mathrm{al}^{14}$ have also reported that LVSI is an independent risk factor for recurrence and poor outcomes in endometrial cancer. Taking those studies into account, it becomes clear that the current case, which highlights the difficulty in distinguishing between lymphovascular involvement and pseudoinvasion, is particularly relevant.

In a review, McCluggage ${ }^{11}$ acknowledged the exceeding difficulty of diagnosing pathological tissue in vascular pseudoinvasion and offered guidelines for its identification. Clues for recognizing vascular pseudoinvasion as a grossing artifact include the contrast between the high volume of vascular invasion and low tumor grade, the presence of stromal tissue alongside the glands within vessels, preferential involvement of large thick-walled blood vessels in the outer myometrium, and the absence of tumor adherence to the vessel wall. Other distinguishing characteristics of their specimens included endometrial lining disruption, as well as endometrial clefts harboring tumor fragments, intratubal contaminants, occurrences of nuclear crush artifact, and the presence of intravascular inflammatory debris. Increased awareness of this artifact, as well as its accurate identification through feature recognition, can safeguard against misinter- 
Table 2.

Criteria for True and Artifactual LSVI

\begin{tabular}{ll}
\hline True LSVI & Pseudo-LSVI \\
\hline $\begin{array}{l}\text { Smooth bordered cohesive } \\
\text { clusters of tumor cells }\end{array}$ & $\begin{array}{l}\text { Disaggregated tumor cells, often } \\
\text { intermixed with inflammation } \\
\text { Clusters conform to the } \\
\text { contours of the }\end{array}$ \\
$\begin{array}{l}\text { lymphovascular space } \\
\text { lymphovascular space }\end{array}$ \\
$\begin{array}{l}\text { Often a change in } \\
\text { morphology with more }\end{array}$ & $\begin{array}{l}\text { Similar morphology to intact } \\
\text { tumor, sometimes as intact } \\
\text { fymphilic cytoplasm } \\
\text { to large vessels }\end{array}$ \\
& $\begin{array}{l}\text { Spaces are immediately adjacent } \\
\text { to invasive tumor with } \\
\text { retraction artifact }\end{array}$
\end{tabular}

Folkins AK et al. Evaluation of vascular space involvement in endometrial adenocarcinomas: Laparoscopic vs abdominal hysterectomies. Modern Pathology 2010; 23(8): $1075^{6}$

pretation that can lead to unnecessary adjuvant treatment later on.

In addition, in a similar effort aimed at simplifying the evaluation of vascular space involvement in endometrial adenocarcinoma, Folkins et al ${ }^{9}$ established biological criteria for distinguishing between true vascular space involvement and pseudo vascular involvement. (Table 2). Furthermore, their consensus report, which was conducted after a review of original case studies included the possibility that LVSI-negative cases could in reality be positive instead, or vice versa, emphasizing the critical nature of accurate distinction.

Regarding the facilitated identification of pseudo vascular invasion, Kitahara et $\mathrm{al}^{8}$ reported that laparoscopic hysterectomy (LH) is associated with a higher rate of vascular pseudoinvasion than laparotomy but that this phenomenon cannot be attributed solely to the mechanical disruption, displacement, and transport of tissue into vascular space that sometimes accompany the use of a uterine manipulator. Although the number of cases was small $(\mathrm{N}=21)$, the same group also reported a significant difference between the rate of true vascular space invasion in LHs (33\%) and total abdominal hysterectomies (TAH) cases $(0 \% ; P=.001)$. Folkins et $\mathrm{al}^{9}$ also noted that laparoscopic procedures tend to have a higher index of vascular involvement that is associated with lower stage and fewer lymph node metastases. On the other hand, in 3 separate studies ${ }^{15-17}$ based on surgery data from a large number of patients from a single institution in whom a single uterine manipulator was used and a pathology review conducted by 2 or more pathologists at a tumor board conference (including the gynecologic pathology specialist), no significant difference in the frequency of LVSI between laparoscopic hysterectomy (LAVH, TLH, and robot-assisted laparoscopy) and laparotomy was reported. In addition, a Gynecologic Oncology Group (GOG) LAP2 Trial study ${ }^{18}$ showed no significant difference in risk of cancer recurrence and overall survival $(\sim 60$ mo) between laparoscopy and laparotomy but did not address LVSI rate for laparoscopy versus laparotomy.

Finally, for cases in the current study in which the Vcare uterine manipulator was used, (pseudo) benign endometrial gland vascular involvement was observed at more than twice the rate of those in which the ClearView uterine manipulator was used. This difference may be attributable to the relative ease with which Vcare allows for the surgeon's preferential positioning of the uterus. The freedom of movement afforded may actually result in the occasional application of excess stress on the uterus. However, because the sample size of 53 cases was relatively small, it is difficult to tell with any certainty whether the observed variation is indeed related to some inherent difference between the 2 uterine manipulators used.

In the current study, the index case represents an example of overtreatment owing to the difficulty in distinguishing between true and pseudoinvasion. Not only that, but our retrospective study demonstrated that pseudoinvasion is present in a significant number of cases, increasing the possibility of overtreatment. Additional reports are expected in the near future, but even now it is extremely important that gynecologists, surgeons, and pathologists refamiliarize themselves with the diagnostic pathology described herein and use it as a reference and point of discussion going forward. The present finding highlights the need to reinstall the careful differentiation of pseudoinvasion from true LVSI as a salient topic of discussion, especially in the present context. Furthermore, the clinical significance of pseudoinvasion remains unclear. Is it implicitly devoid of the potential to be a risk factor or not? At present, this question remains unanswered and warrants further investigation. It is still possible that, in some cases, true LVSI may be inadvertently treated as pseudoinvasion, resulting, in undertreatment of a serious condition. Through careful differentiation of true invasion from pseudoinvasion and ongoing research to determine the clinical significance of pseudoinvasion, we can mitigate both undertreatment and overtreatment.

The authors thank Mr. Akira Matsumoto, CT, and Mr. Shota Otomo, CT, from the Kokura Medical Center Department of Pathology for technical assistance. 


\section{References:}

1. Walker JL, Piedmonte MR, Spirtos NM, et al. Laparoscopy compared with laparotomy for comprehensive surgical staging of uterine cancer: Gynecologic Oncology Group Study LAP2. J Clin Oncol. 2009;27:5331-5336.

2. Mourits MJ, Bijen CB, Arts HJ, et al. Safety of laparoscopy versus laparotomy in early-stage endometrial cancer: a randomized trial. Lancet Oncol. 2010;11:763-771.

3. Janda M, Gebski V, Brand A. Quality of life after total laparoscopic hysterectomy versus total abdominal hysterectomy for stage I endometrial cancer (LACE): a randomised trial. Lancet Oncol. 2010;11:772-780.

4. Nagao S, Fujiwara K, Kagawa R, et al. Feasibility of extraperitoneal laparoscopic para-aortic and common iliac lymphadenectomy. Gynecol Oncol. 2006;103:732-735.

5. Fujimoto T, Fukuda J, Tanaka T. Role of complete paraaortic lymphadenectomy in endometrial cancer. Curr Opin Obstet Gynecol. 2009;21:10-14.

6. Terai Y, Tanaka T, Sasaki H, et al. Total laparoscopic modified radical hysterectomy with lymphadenectomy for endometrial cancer compared with laparotomy. J Obstet Gynecol Res. 2014;40:570-575.

7. Logani S, Herdman AV, Little JV, Moller KA. Vascular "pseudo invasion" in laparoscopic hysterectomy specimens: a diagnostic pitfall. Am J Surg Pathol. 2008;32:560-565.

8. Kitahara S, Walsh C, Frumovitz M, Malpica A, Silva EG. Vascular pseudo invasion in laparoscopic hysterectomy specimens for endometrial carcinoma: a grossing artifact? Am J Surg Pathol. 2009;33:298-303.

9. Folkins AK, Nevadunsky NS, Saleemuddin A, et al. Evaluation of vascular space involvement in endometrial adenocarcinomas: laparoscopic vs abdominal hysterectomies. Modern Pathol. 2010;23:1073-1079.

10. Krizova A, Clarke BA, Bernardini MQ, et al. Histologic artifact in abdominal, vaginal, laparoscopic, and robotic hysterec- tomy specimens: a blinded retrospective review. Am J Surg Pathol. 2011;35:115-126.

11. McCluggage WG. Ten problematical issues identified by pathology review for multidisciplinary gynaecological oncology meetings. J Clin Pathol. 2012;65:293-301.

12. Weinberg LE, Kunos CA, Zanotti KM. Lymphovascular space invasion (LVSI) is an isolated poor prognostic factor for recurrence and survival among women with intermediate- to high-risk early-stage endometrioid endometrial cancer. Int J Gynecol Cancer. 2013;23:1438-1445.

13. Guntupalli SR, Zighelboim I, Kizer NT, et al. Lymphovascular space invasion is an independent risk factor for nodal disease and poor outcomes in endometrioid endometrial cancer. Gynecol Oncol. 2012;124:31-35.

14. Nofech-Mozes S, Ackerman I, Ghorab Z, et al. Lymphovascular invasion is a significant predictor for distant recurrence in patients with early-stage endometrial endometrioid adenocarcinoma. Am J Clin Pathol. 2008;129:912-917.

15. Momeni M, Kolev V, Cardenas-Goicoechea J, et al. Does the type of surgery for early-stage endometrial cancer affect the rate of reported lymphovascular space invasion in final pathology specimens? Am J Obstet Gynecol. 2013;208:71.e1-e6.

16. Hopkins MR, Richmond AM, Cheng G, et al. Lymphovascular space invasion in robotic surgery for endometrial cancer. JSLS. 2014;18:e2014.00021.

17. Zhang C, Havrilesky LJ, Broadwater G, et al. Relationship between minimally invasive hysterectomy, pelvic cytology, and lymph vascular space invasion: a single institution study of 458 patients. Gynecol Oncol. 2014;133:211-215.

18. Walker JL, Piedmonte MR, Spirtos NM, et al. Recurrence and survival after random assignment to laparoscopy versus laparotomy for comprehensive surgical staging of uterine cancer: Gynecologic Oncology Group LAP2 Study. J Clin Oncol. 2012;30: 695-700. 